\title{
Descrição das fêmeas de Chimarra (Curgia) brasiliana (Ulmer) e Chimarra (Curgia) ypsilon Flint (Insecta, Trichoptera, Philopotamidae) do Estado do Paraná, Brasil ${ }^{1}$
}

\author{
Gisele Luziane de Almeida ${ }^{2,3}$ \\ Luciane Marinoni ${ }^{2,4}$
}

\begin{abstract}
Description of the females of Chimarra (Curgia) brasiliana (Ulmer) and Chimarra (Curgia) ypsilon Flint (Insecta, Trichoptera, Philopotamidae) from Paraná State, Brazil. Mating couples of two species of the genus Chimarra Stephens, C. (Curgia) brasiliana (Ulmer, 1905) and C. (Curgia) ypsilon Flint, 1983, were collected with light trap at Paraná State, Brazil. The females are described and illustrations of the genitalia are presented.

KEY WORDS. Trichoptera, Philopotamidae, females, light trap, Chimarra, Paraná, Brazil
\end{abstract}

Muitas espécies de Trichoptera são conhecidas apenas por exemplares machos, o que tem dificultado um entendimento taxonômico mais amplo do grupo. No entanto, algumas espécies têm suas fêmeas identificadas através do método de associação, o qual se baseia principalmente na coloração geral dos exemplares. Este procedimento não é tão preciso quanto a captura de casais em cópula ou a criação de imaturos para obtenção dos adultos, já que fềmeas de determinados grupos possuem morfologia externa muito semelhante (BLAHNIK 1997, 1998; COOPER \& MORSE 1998).

Uma dificuldade ainda maior é encontrada quando levantamentos da fauna de Trichoptera são realizados com auxílio de armadilhas luminosas. Alguns autores têm demonstrado que a maioria dos exemplares capturados através desta metodologia é de fêmeas e, que associação entre machos e fêmeas torna-se ainda mais difícil pelo fato dos exemplares serem preservados em álcool, o que leva à perda do padrão de coloração, caráter importante na identificação (MCELRAVY et al. 1981; MARINONI \& ALMEIDA 2000; ALMEIDA \& MARINONI 2000). É evidente também que, se todas as fêmeas pudessem ser identificadas até o nível de espécie, determinadas conclusões, principalmente aquelas embasadas em índices ecológicos de diversidade, poderiam ser mais acuradas (GUEROLD 2000).

$\mathrm{O}$ estudo dos Trichoptera provenientes do levantamento entomofaunístico conduzido em oito localidades do Estado do Paraná - PROFAUPAR (MARINONI \& DUTRA 1993) possibilitou o registro de dois casais em cópula, um pertencente à espécie Chimarra (Curgia) brasiliana (Ulmer, 1905) e outro à Chimarra (Curgia) ypsilon Flint, 1983 (Philopotamidade, Chimarrinae) (Figs 1-4).

1) Contribuição número 1273 do Departamento de Zoologia, Universidade Federal do Paraná.

2) Departamento de Zoologia, Universidade Federal do Paraná. Caixa Postal 19020, 81531-990 Curitiba, Paraná, Brasil.

3) Bolsista da CAPES. E-mail: giseleluziane@yahoo.com

4) Pesquisador do CNPq. E-mail Imarinon@bio.ufpr.br 

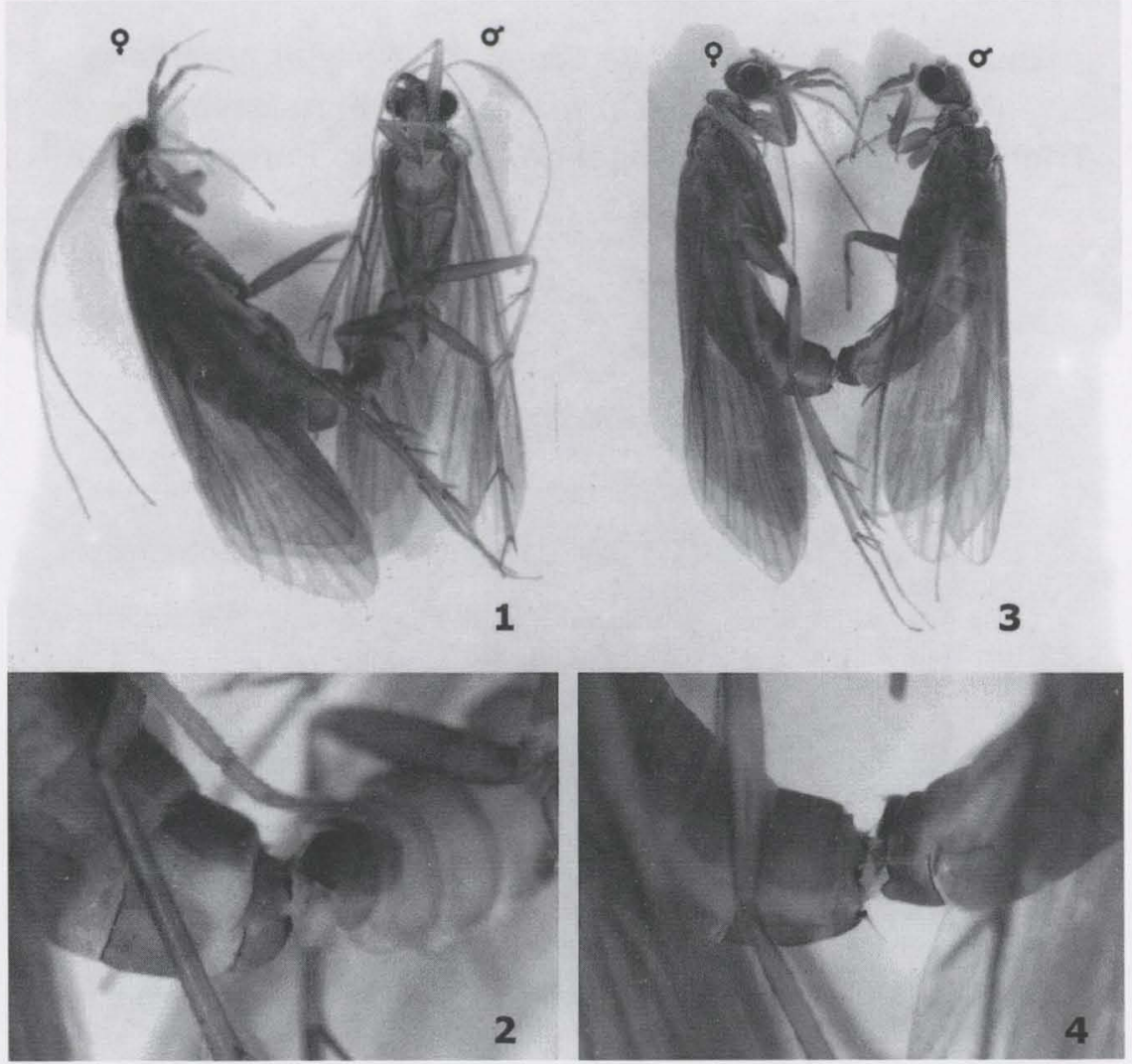

Figs 1-4. Casais em cópula. (1-2) Chimarra (Curgia) brasiliana; (3-4) Chimarra (Curgia) ypsilon.

O gênero Chimarra Stephens, 1829 é amplamente distribuído pelas regiões tropicais e subtropicais do continente americano e, dentre os gêneros de Philopotamidae, é um dos maiores em número de espécies (BLAHNIK 1997, 1998; COOPER \& MORSE 1998; FLINT 1981, 1983, 1991; FLINT et al. 1999). Atualmente são conhecidas cerca de 400 espécies, das quais 222 possuem registros para a região Neotropical (FLINT et al. 1999). Espera-se ainda um acréscimo aproximado de $50 \%$ na riqueza de espécies de Chimarra com a intensificação de levantamentos entomofaunísticos e de estudos taxonômicos com o grupo (BLAHNIK 1997, 1998; FLINT et al. 1999).

De acordo com Flint (1998), o subgênero C. (Curgia) Walker, 1860 é definido por variações em caracteres externos como coloração do corpo, asas e apêndices e por modificações nas garras tarsais das pernas anteriores dos machos, porém, as espécies são basicamente descritas por caracteres de genitália masculina. Das 92 espécies reconhecidas para este subgênero, somente $C$. (Curgia) morio (Burmeister, 1839) teve a genitália da fêmea estudada, pois a descrição original foi realizada com base em fềmeas. 
Segundo Ross (1956), há caracteres taxonômicos importantes na genitália das fêmeas de Chimarra que podem suportar a monofilia do gênero. Da mesma forma, FLINT (1998) menciona que a presença de um processo no oitavo esternito da fêmea é possivelmente uma sinapomorfia para o subgênero Curgia, havendo necessidade, no entanto, de mais estudos relacionados à filogenia do grupo.

Diante da falta de informações relacionadas à taxonomia de fềmeas de Trichoptera e da importância do seu conhecimento, os objetivos deste trabalho são descrever as genitálias das fêmeas das espécies capturadas em cópula, Chimarra (Curgia) brasiliana e Chimarra (Curgia) ypsilon, e apresentar ilustrações de suas genitálias.

\section{MATERIAL E MÉTODOS}

Os espécimes utilizados neste estudo foram capturados com o auxílio de armadilha luminosa durante o Levantamento da Fauna Entomológica no Estado do Paraná - PROFAUPAR (MARINONI \& DUTRA 1993; MARINONI \& ALMEIDA 2000; AlmEIDA \& MARINONI 2000). Os dois casais em cópula, um de C. (Curgia) brasiliana e outro de C. (Curgia) ypsilon, foram capturados na localidade de Guarapuava $\left(25^{\circ} 40^{\prime} \mathrm{S}, 52^{\circ} 01^{\prime} \mathrm{W}\right)$.

A identificação dos exemplares machos foi feita utilizando-se a chave de FLINT (1998) para espécies do subgênero Curgia. Após este procedimento, outras fêmeas capturadas na mesma localidade foram identificadas e, posteriormente, utilizadas na confecção dos desenhos. Também foram identificadas fêmeas das referidas espécies capturadas no Estado do Paraná, Brasil, nas localidades de Jundiaí do Sul, Fênix, Telêmaco Borba, Ponta Grossa e Antonina.

As ilustrações das genitálias foram realizadas com auxílio de câmara clara acoplada a estereomicroscópio Wild M-5 e a terminologia adotada para caracterizar as estruturas foi a de NIELSEN (1980).

Para ilustrar a morfologia externa das genitálias das fêmeas, foram utilizados exemplares preservados em álcool a $70 \%$. Os exemplares examinados encontram-se depositados na Coleção de Entomologia Pe. Jesus S. Moure, Departamento de Zoologia, Universidade Federal do Paraná, Curitiba, Brasil (DZUP).

\section{RESULTADOS E DISCUSSÃO}

\section{Chimarra (Curgia) brasiliana (UImer, 1905)}

Fig. $5 a, b$

Chimarrha brasiliana Ulmer, 1905a: 96; Tomaszewski, 1961: 2.

Wormaldia parva Ulmer, 1905b: 90; Flint, 1966: 3.

Chimarra brasiliana (Ulmer); Fischer, 1961: 58; Flint, 1966: 3.

Chimarra parva (Ulmer); Fischer, 1961: 68.

Chimarra (Curgia) brasiliana (Ulmer); Flint, 1972: 228; 1998: 72; Flint et al., 1999: 150.

Descrição. Genitália da fêmea. Tergito e esternito VIII fusionados, formando um anel esclerotinizado; ventralmente com um processo mediano curto, afilado no ápice; margem posterior recortada tendo a cada lado três lóbulos enegrecidos, de forma irregular, com cerdas longas; médio-ventralmente com emarginação trunca- 
da. Tergito IX esclerotinizado, com uma fileira de cerdas ligeiramente linear próxima à margem posterior. Esternito IX pequeno, algo cordiforme, mais esclerotinizado posteriormente. Tergito X representado por um par de lobos, pouco esclerotinizados, cada um com um pequeno cerco apical.

Material examinado. BRASIL, Paraná: Guarapuava, $25^{\circ} 40^{\prime} \mathrm{S}, 52^{\circ} 01^{\prime} \mathrm{W}, 740$ m, 28.I.1987, PROFAUPAR leg., 1 casal em cópula e 16 fêmeas; Jundiaí do Sul,

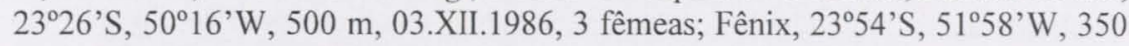
m, 29.XI.1986, 1 fềmea; Telêmaco Borba, 24¹7'S, 50³7' W, 750 m, 01.XI.1986, 1 fềmea; Ponta Grossa, 25 $5^{\circ} 14^{\prime} \mathrm{S}, 50^{\circ} 03^{\prime} \mathrm{W}, 880$ m, 27.I.1987, 5 fềmeas; Antonina, $25^{\circ} 28^{\prime}$ 'S, 4850'W, 60 m, 01.III.1987, 51 fềmeas.

Distribuição. Brasil (Paraná e Santa Catarina), Paraguai, Argentina e Uruguai.

Comentários. As espécies de C. (Curgia) estão reunidas em diferentes grupos com base principalmente nas similaridades dos caracteres das genitálias masculinas (FLINT 1998). Chimarra (Curgia) brasiliana está incluída no grupo banksi por compartilhar com outras 28 espécies os seguintes caracteres: presença de escova de pêlos e outros processos no tergito VIII; tergito IX com placa dorsal desenvolvida; redução do número de espinhos do falo de 4 a 1 ou totalmente ausentes e redução do clásper. O grupo banksi, por sua vez, está dividido em 11 complexos de espécies, sendo que $C$. (C.) brasiliana juntamente com $C$. (C.) piraya Flint, 1983, C. (C.) cultellata Flint, 1983, C. (C.) parana Flint, 1972 e C. (C.) fittkaui Flint, 1971 formam o complexo brasiliana. As espécies deste complexo são bastante semelhantes externamente. Tergito VIII com um único e alongado lóbulo póstero-lateral com uma escova de pêlos; placa dorsal do tergito IX com um lóbulo especializado, também possuindo uma pequena escova de pêlos de forma variada. Outras características como coloração de asas, corpo e apêndices, bem como modificações de garras tarsais, também são consideradas. Os caracteres que definem a espécie C. (C.) brasiliana são: tíbia e tarso de coloração escura, antenas pálidas; cabeça e tórax, dorsalmente com pêlos brancos; asas anteriores escuras com máculas branco-prateadas espalhadas; garras tarsais das pernas anteriores dos machos aparentemente não modificadas. Os exemplares conservados em álcool apresentam coloração castanha.

\section{Chimarra (Curgia) ypsilon Flint, 1983}

Fig. $6 a, b$

Chimarra (Curgia) ypsilon Flint, 1983: 16; 1998: 49; Flint et al., 1999: 162.

Descrição. Genitália da fêmea. Tergito e esternito VIII fusionados, formando um anel esclerotinizado; ventralmente com um processo mediano curto, arredondado no ápice; margem posterior recortada tendo a cada lado três lóbulos enegrecidos com cerdas longas; médio-ventralmente com emarginação angulosa. Tergito IX esclerotinizado, com uma fileira de cerdas ligeiramente linear próxima à margem posterior. Esternito IX losangular com duas áreas subtriangulares basais mais esclerotinizadas. Tergito X representado por um par de lobos, pouco esclerotinizados, cada um com um pequeno cerco apical. 


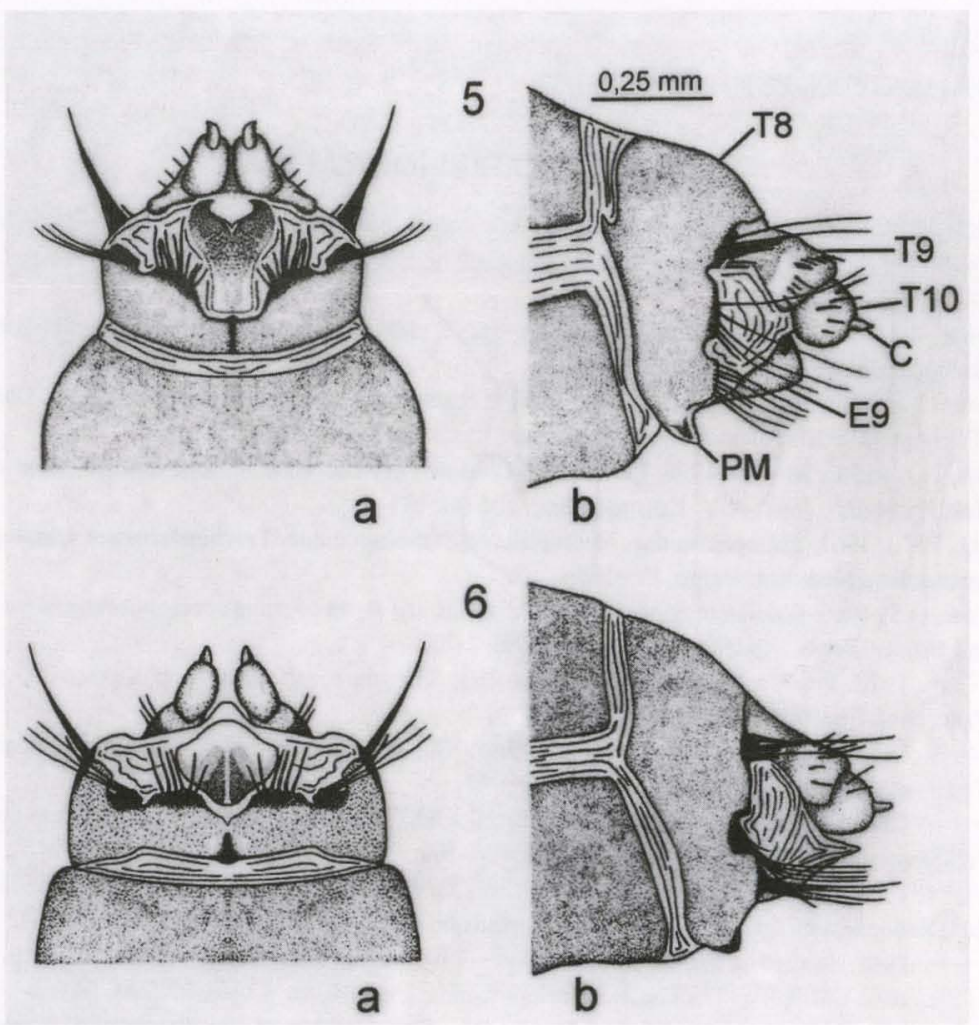

Figs 5-6. Genitália feminina. (5) Chimarra (Curgia) brasiliana; (6) Chimarra (Curgia) ypsilon: (a) vista ventral, (b) vista lateral. (T) Tergito, (E) esternito, (PM) processo mediano do esternito VIII, (C) cerco.

Material examinado. BRASIL, Paraná: Guarapuava, $25^{\circ} 40^{\prime} \mathrm{S}, 52^{\circ} 01^{\prime} \mathrm{W}, 740$ m, 28.I.1987, PROFAUPAR leg., 1 casal em cópula, 12 fêmeas; idem, 27.III.1987, 10 fêmeas.

Distribuição. Brasil (Minas Gerais, Rio de Janeiro, Paraná e Santa Catarina), Paraguai e Argentina.

Comentários. Segundo Flint (1998), C. (Curgia) ypsilon está incluída no grupo mexicana, cujos integrantes compartilham as seguintes características gerais: corpo de coloração castanha, apêndices geralmente pálidos; cabeça e tórax podendo estar cobertos com cerdas douradas; asas anteriores castanhas, geralmente com manchas ou linhas douradas; ausência de uma escova de pêlos no tergito VIII. Chimarra (Curgia) ypsilon possui como características próprias: asas anteriores amplamente marcadas com pêlos dourados, especialmente na base, e com uma marca em forma de Y na margem costal do estigma; garras das pernas anteriores dos machos extremamente assimétricas. As fềmeas preservadas em álcool têm coloração castanha. 
AGRADECIMENTOS. Somos gratas a Marcelo Duarte, Renato C. Marinoni e Sionei Ricardo

Bonatto pela leitura crítica e sugestões.

\section{REFERÊNCIAS BIBLIOGRÁFICAS}

AlmeidA, G.L. \& L. MArinONI. 2000. Abundância e sazonalidade das espécies de Leptoceridae (Insecta, Trichoptera) capturadas com armadilha luminosa no Estado do Paraná, Brasil. Revta bras. Zool. 17 (2): 347-359.

BLAHNIK, R.J. 1997. Systematics of Chimarrita, a new subgenus of Chimarra (Trichoptera: Philopotamidae). Syst. Ent. 22: 199-243.

-1998. A revision of the neotropical species of the genus Chimarra, subgenus Chimarra (Trichoptera: Philopotamidae). Mem. Amer. Ent. Inst. 59: 1-318.

COOPER, H.J. \& J.C. MoRSE. 1998. Females of Chimarra (Trichoptera: Philopotamidae) from eastern North America. Jour. N.Y. Entomol. Soc. 106 (4): 185-198.

FISCHER, F.C.J. 1961. Philopotamidae, Hydroptilidae, Stenopsychidae. Trichopterorum Catalogus II. Amsterdam, Ned. Ent. Veren. IV+189p.

FLINT JR., O.S. 1966. Studies of neotropical caddis flies, III: types of some species described by Ulmer and Brauer. Proc. U.S. Nat. Mus. 120 (3559): 1-20.

1972. Studies of neotropical caddis flies, XIV: on a collection from northern Argentina.

Proc. Biol. Soc. Wash. 85 (17): 223-248.

- 1981. Studies of neotropical caddisflies, XXVIII: the Trichoptera of the Río Limón Basin, Venezuela. Smithson. Contrib. Zool. 330: 1-61.

1983. Studies of neotropical caddisflies, XXXII: new species from Austral South America (Trichoptera). Smithson. Contrib. Zool. 377: 1-100.

-1991. Studies of neotropical caddisflies, XLV: the taxonomy, phenology, and faunistics of the Trichoptera of Antioquia, Colombia. Smithson. Contrib. Zool. 520: 1-113.

-1998. Studies of neotropical caddisflies, LIII: a taxonomic revision of the subgenus Curgia of the genus Chimarra (Trichoptera: Philopotamidae). Smithson. Contrib. Zool. 594: 1-130.

Flint JR., O.S.; R.W. HolzenthAl \& S.C. HARris. 1999. Catalog of the Neotropical Caddisflies (Insecta: Trichoptera). Columbus, Ohio Biological Survey, IV+239p.

GUerold, F. 2000. Influence of taxonomic determination level on several community indices. Wat. Res. 34 (2): 487-492.

Marinoni, L. \& G.L. Almeida. 2000. Abundância e sazonalidade das espécies de Hydropsychidae (Insecta, Trichoptera) capturadas em armadilha luminosa no Estado do Paraná, Brasil. Revta bras. Zool. 17 (1): 283-299.

MARINONI, R.C. \& R.R.C. Dutra. 1993. Levantamento da fauna entomológica no Estado do Paraná. I. Introdução. Situações climáticas e florísticas de oito pontos de coleta. Dados faunísticos de Agosto de 1986 a Julho de 1987. Revta bras. Zool. 8 (1/2/3/4): 31-73.

McElravy, E.P.; V.H. Resh; H. Wolda \& O.S. Flint JR. 1981. Diversity of adult Trichoptera in a non-seasonal tropical environment. Proc. $3^{\text {rd }}$ Int. Symp. Trichoptera 20: 149-156.

NIELSEN, A. 1980. A comparative study of the genital segments and the genital chamber in female Trichoptera. Copenhagen, Det Kongelige Danske Videnskabernes Selskab, 200p.

Ross, H.H. 1956. Evolution and classification of the mountain caddisflies. Urbana, Univ. Illinois Press, 213p.

TOMASZEWISKI. 1961. List of the specimens in the Collection of the Institute of Zoology, of the Polish Academy of Sciences in Warsaw, IV: caddis flies (Trichoptera). Ann. Zool. (Wars) 20: 1-6.

UlmeR, G. 1905a. Zur Kenntniss aussereuropäischer Trichopteren. Stett. Entomol. Zeit. 66: 1-119.

-1905b. Neue und wenig bekannte aussereuropäische Trichopteren, hauptsächlich aus dem Wiener Museum. Ann. k.k. naturhis. Hofmuseums 20: 59-98.

Recebido em 10.XII.2000; aceito em 28.VIII. 2001. 